\title{
Geometrische Bestimmung des
}

\section{Krümmungsmittelpunktes der algebraischen Spiralen.}

\author{
Von Wilhelm Rulf in Wien.
}

Die Polarsubnormalen der algebraischen Spiralen, deren Abhängigkeit von den Constanten. und Polarcoordinaten man bekanntlich mittelst ähnlicher Dreiecke - also auf elementar-geometrisehem Wege - ermitteln kann, kann man als Radiivectores einer neven Spirale ansehen, welche im folgenden die Polarsubnormalen-Spirale oder kurz Subnormalen-Spirale der gegebenen Spirale genannt werden soll.

Es sei Fig. 1. $O$ der Pol, $P$ ein Punkt der algebraischen Spirale, $T$ die Tangente in demselben, $N$ die Normale und demnach, wenn $R Q$ senkrecht auf $O P$ steht, $O Q$ die Polarsubnormale und $O R$ die Polarsubtangente.

Um den Krümmungsmittelpunkt $M$ zu finden, müsste man bekanntlich die unendlich nahe Normale $N^{\prime}$ mit $N$ zum Schnitt bringen. Der unendlich nahe Punkt $P^{\prime}$ der Spirale, dessen Normale $N^{\prime}$ ist, liegt aber auch in der Tangente $T$ und der zugehörige Punkt $Q^{\prime}$, der Endpunkt der Polarsubnormale, in der Tangente $t$ der Subnormalenspirale. Da aber auch $P^{\prime} O Q^{\prime}$ ein rechter Winkel ist, so ist nach einem bekannten Satze der projectiven Geometrie $N^{\prime}$ die Tangente eines Kegelschnittes, der $T, t$ und $N$ berührt, und demnach der Krümmungsmittelpunkt als Schnittpunkt der zwei unendlich nahen Kegelschnitttangenten $N N^{\prime}$ der Be-

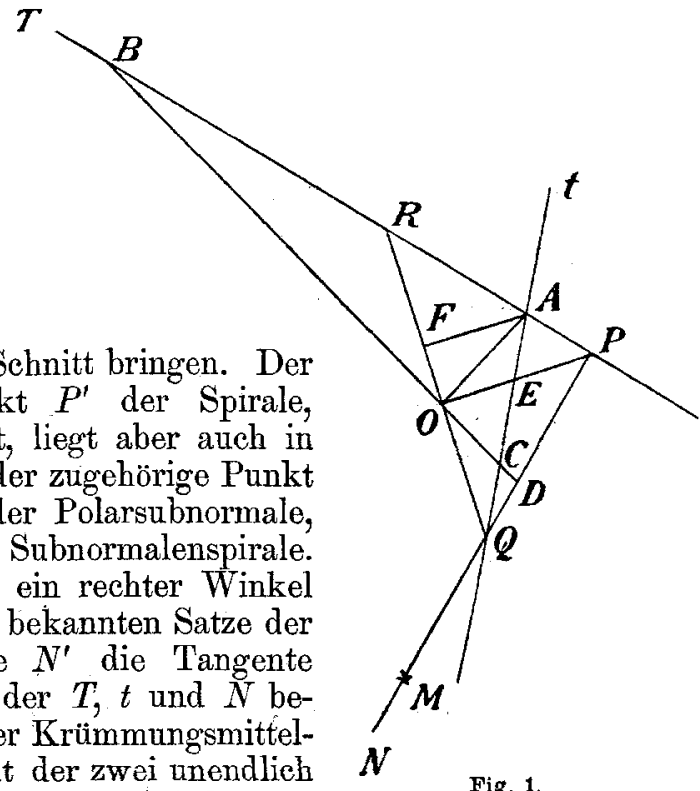

Fig. 1 . rührungspunkt von $N$. Daher ist er der vierte harmonische Punkt zu $D$ in Bezug auf $Q$ und $P$, wenn $D$ der Schnittpunkt der Berührungssehne $B C$ der beiden Tangenten $T$ und $t$ mit $N$ ist. Diese Berührungssehne steht aber. bekanntlich auf $O A$ senkrecht, wenn mit $A$ der Schnittpunkt der beiden Tangenten $T$ und $t$ bezeichnet wird. 
Hiemit ist der Krümmungsmittelpunkt geometrisch gefunden, wenn noch gezeigt wird, dass die Tangente $t$ der Subnormalenspirale geometrisch, ohne Benützung der Differentialrechnung gefunden werden kann.

Soll der Krümmungsradius $P M$, der in der Folge immer mit $x$ bezeichnet wird, berechnet werden, so benütze man die harmonischen Punkte $M Q D$ und $P$, vermöge welcher ohne Rücksicht auf die Vorzeichen der Strecken die Gleichung besteht:

$$
\left.\frac{x}{x-P Q}=\frac{P D}{P Q-P D} \cdots \cdots 1\right),
$$

worin $P Q$ die Normale und aufer $x$ nur $P D$ unbekannt ist.

$\mathrm{Da}$ aber wegen paarweise normaler Seiten $\triangle O D P$ ähnlich $\triangle O A R$ ist, so besteht die Proportion:

$$
\begin{gathered}
P D: A R=r: O R \\
\text { and } \left.\quad P D=\frac{r \cdot A R}{O R} \cdots \cdot 2\right)
\end{gathered}
$$

worin $O R$ die Polar-Subtangente ist und $A R$ aus den Beziehungen der Tangente $t$ berechnet werden muss.

Das hier im allgemeinen Gezeigte soll nun insbesondere an folgenden algebraischen Spiralen durchgeführt werden: 1 . An der Neoide, Polargleichung $r=a \varphi+b ; 2$ an der reciproken oder hyperbolischen spirale, $r=\frac{a}{\varphi}$; 3 . an der parabolischen (Fermat'sche) Spirale $r^{2}=a^{2} \varphi$ und 4. an der reciproken parabolischen Spirale (Lituus) $r^{2}=\frac{a}{\varphi}$.

1. Krümmungsmittelpunkt der Neoide.

Bei dieser ist bekanntlich die Subnormalen-Spirale ein Kreis, daher $t$ senkrecht auf $O Q$.

$\mathrm{Da} Q A \| O P$, so findet die Proportion statt

$$
A R: P R=Q R: O R \text {. }
$$

Darin ist $P R=\frac{r\left(a^{2}+r^{2}\right)^{\frac{1}{2}}}{a}, Q R=\frac{a^{2}+r^{2}}{a}, O R=\frac{r^{2}}{a}$, daher

und mittelst 2)

$$
A R=\frac{\left(a^{2}+r^{2}\right)^{\frac{3}{2}}}{a r}
$$

$$
P D=\frac{\left(a^{2}+r^{2}\right)^{\frac{3}{2}}}{r^{2}}
$$


und 1)

$$
x=\frac{\left(a^{2}+r^{2}\right)^{\frac{3}{2}}}{2 a^{2}+r^{2}},
$$

die bekannte Formel für den Krümmungshalbmesser der Neoide.

2. Der Krümmungsmittelpunkt der hyperbolisehen Spirale.

Die Polarsubnormale derselben ist, wie sich leicht mittelst ähnlicher Dreiecke zeigen lässt, $\frac{r^{2}}{a}=\frac{a}{0^{2}}$. Bezeichnet man dieselbe mit $\rho$, so lautet die Gleichung der Subnormalen-Spirale:

$$
\rho=\frac{a}{\varphi^{2}} \text {. }
$$

Zur Ermittelung ihrer Tangente $t$ wird ihre Polarsubtangente mittelst Fig. 2 berechnet.

Es sei Fig $2 O$ der Pol, $P$ irgend ein Punkt dieser Spirale. Für den unendlich nahen Punkt $P^{\prime}$ derselben ist der Leitstrahl $O P^{\prime}=p_{1}$ kleiner als $O P=P$, weil vermöge der Gleichung der Leitstrahl mit wachsendem Winkel o abnimmt. Die Verbindungslinie $P P^{\prime}$ ist die verlangte Tangente $t$, und wenn $O A$ senkrecht steht auf $O P$, so ist $O A$

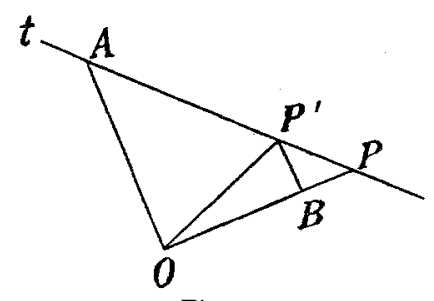

Fig. 2. die Polarsubtangente.

Beschreibt man mit $p_{1}$ den Kreisbogen $P^{\prime} B$, so kann man diesen als gerade und parallel zu $O A$ betrachten, weshalb die Proportion stattfindet:

$$
O A: P^{\prime} B=O P: B P
$$

worin $P^{\prime} B=p_{1}\left(\varphi_{1}-\varphi\right), O P=p$ and $B P=\rho-p_{1}$.

Ersetzt man überall die Leitstrahlen durch die Winkel, so erhält man nach gehöriger Reduction

$$
O B=\frac{a}{2 \varphi} \text {. }
$$

$\frac{a}{\varphi}$ ist aber der Leitstrahl der hyperbolischen Spirale, daher geht die Tangente ihrer Subnormalen-Spirale durch den Mittelpunkt des Leitstrahles. Die Tangente $t$ wird daher in Fig. 1 dadurch gefunden, dass man $O P$ in $E$ halbiert, und $E$ mit $Q$ verbindet.

Um aus dieser Figur $A R$ (siehe Gleichung 2) zu berechnen, fálle man von $A$ auf $Q R$ die Senkrechte $A F$, bezeichne dieselbe 
mit $y, F Q$ mit $z$ und $O R$ mit $m$. Dann ist $O Q=\frac{r^{2}}{m}, Q R=\frac{m^{2}+r^{2}}{m}$ und weil $\dot{A} F \| O P$

$$
\begin{gathered}
y: z=\frac{r}{2}: \frac{r^{2}}{m} \\
y:\left(\frac{m^{2}+r^{2}}{m}-z\right)=r: m .
\end{gathered}
$$

Aus diesen Proportionen folgt:

und endlich

$$
\begin{aligned}
& y=\frac{r\left(m^{2}+r^{2}\right)}{m^{2}+2 r^{2}} \\
& z=\frac{2 r^{2}\left(m^{2}+r^{2}\right)}{m\left(m^{2}+2 r^{2}\right)}
\end{aligned}
$$

$$
A R=\sqrt{y^{2}+z^{2}}=\frac{\left(m^{2}+r^{2}\right)^{\frac{3}{2}}}{m^{2}+2 r^{2}} .
$$

Anmerkung. In Fig. 1 ist $A P=P R-A R=\frac{r^{2}\left(m^{2}+r^{2}\right)^{\frac{1}{2}}}{m^{2}+2 r^{2}}$, daher $A R: A P=\left(m^{2}+r^{2}\right): r^{2}=P R^{2}: O P^{2}$, d. h.: Verbindet man die Mitte der Hypotenusenhöhe mit einer spitzen Ecke des rechtwinkeligen Dreieckes, so entstehen auf der gegenüberliegenden Kathete zwei Abschnitte, die sich wie das Quadrat über der Kathete zum Quadrat über der Hypotenuse verhalten.

Setzt man den gefundenen Wert für $A R$ in die Gleichung (1), so erhält man, da $P Q=\frac{r}{a}\left(a^{2}+r^{2}\right)^{\frac{1}{2}}$ und $m=a$ ist:

$$
x=r\left(\frac{a^{2}+r^{2}}{a^{2}}\right)^{\frac{3}{2}} \text {. }
$$

3. Der Krümmungsmittelpunkt derparabolischen Spirale. Gleichung ihrer Subnormalen-Spirale:

Die Polarsubnormale derselben ist $\frac{a^{2}}{2 r}=\frac{a}{2 \sqrt{\varphi}}$, daher die

$$
p=\frac{a}{2 \sqrt{\varphi}} \text {. }
$$

Da mit wachsendem $\varphi$ der Leitstrahl $\rho$ abnimmt, so kann zur Berechnung der Polarsubtangente dieser Curve auch Fig. 2 benützt werden. Aus dieser folgt in ähnlicher Weise wie früher:

$$
O B=\frac{a\left(\sqrt{\varphi_{1}}+\sqrt{\varphi}\right)}{2}=a \sqrt{\varphi} .
$$

Aus der Gleichung der parabolischen Spirale folgt aber $V_{\varphi}=\frac{r}{a}$, daher $O B=r$, d. h.: Die Subtangente der Subnormalen- 
Spirale der Fermat'schen Spirale ist gleich dem zugehörigen Leitstrahl der letzteren, und man erkennt leicht, dass dieselbe nicht auf dem Leitstrahl selbst, sondern auf dessen Verlängerung über den Pol $O$ gelegen ist.

Macht man daher in Fig. $3 O E=O P$, so ist die Verbindungslinie $Q E$ die Tangente $t$, und in ähnlicher Weise, wie bei der hyperbolischen Spirale lässt sich zeigen, da

$$
\begin{aligned}
& O R=\frac{2 r^{3}}{a^{2}}, \text { dass } \\
& A R=\frac{r\left(a^{4}+4 r^{4}\right)^{\frac{3}{2}}}{a^{2}\left(a^{4}-4 r^{4}\right)},
\end{aligned}
$$

was auch zu einer ähnlichen Anmerkung wie dort Veranlassung geben könnte.

Endlich ist mittelst 2)

$$
P D=\frac{\left(a^{4}+4 r^{4}\right)^{\frac{3}{2}}}{2 r\left(a^{4}-4 r^{4}\right)},
$$

und mittelst 1)

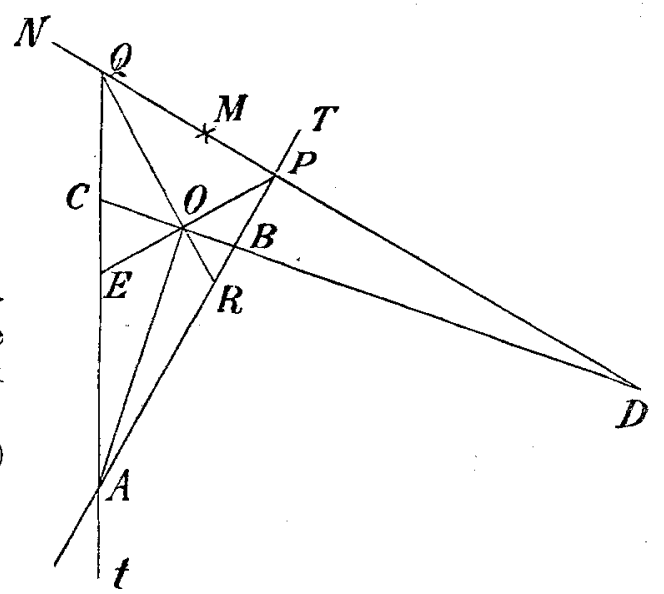

Fig. 3.

$$
x=\frac{r\left(1+4 \varphi^{2}\right)^{\frac{3}{2}}}{2 \varphi\left(3+4 \varphi^{2}\right)},
$$

und wenn die Normale $P Q=N$ eingeführt wird:

$$
x=\frac{N^{3}}{3 N^{2}-2 r^{2}} \text {. }
$$

4. Der Krümmungsmittelpunkt der reciproken parabolischen Spirale.

Die Polarsubnormale derselben ist $\frac{r^{2}}{2 a^{2}}=\frac{a}{2 \varphi \sqrt{\varphi}}$, daher die Gleichung ihrer Subnormalenspirale

$$
\rho=\frac{a}{2 \varphi \sqrt{\varphi}}
$$

und da mit wachsendem $\varphi$ der Leitstrahl $\rho$ abnimmt, so kann man auch zur Berechnung der Subtangente wieder Fig. 2 benützen. Aus dieser folgt durch Einführung der Anomalie und Reduction

$$
O B=\frac{a\left(\varphi_{1}-\varphi\right)}{2\left(\varphi_{1} \sqrt{\varphi_{1}}-\varphi \sqrt{\varphi}\right)}
$$


und wenn man Zähler und Nenner durch $\varphi_{1}-\varphi$ dividiert:

$$
O B=\frac{a}{2\left(\sqrt{\varphi_{1}}+\frac{\varphi\left(\sqrt{\varphi_{1}}-\sqrt{\varphi}\right)}{\varphi_{1}}-\varphi\right.}=\frac{a}{2\left(\sqrt{\varphi_{1}}+\frac{\varphi}{\sqrt{\varphi_{1}}+\sqrt{\varphi}}\right)}
$$

und, wenn $\operatorname{man} \varphi_{1}=\varphi$ setzt

$$
O B=\frac{a}{3 \sqrt{\varphi}}
$$

Da aber der Leitstrahl der reciprocken, parabolischen Spirale gleich ist $\frac{a}{\sqrt{\varphi}}$, so ist auch

$$
O B=\frac{r}{3} \text {. }
$$

Die Polarsubtangente der Subnormalenspirale der reciproken parabolischen Spirale ist demnach $\frac{1}{3}$ vom Leitstrahl der letzteren, und, wie man sich leicht überzeugt, auf demselben selbst gelegen. Man kann daher zur Berechnung des Krümmungshalbmessers Fig. 1 benützen, wenn man sich vorstellet, dass $O E$ ein Drittel von $O P$ ist.

Für die vorliegende Spirale ist

$$
O R=\frac{2 a^{2}}{r}, P Q=\frac{r\left(4 a^{4}+r^{4}\right)^{\frac{1}{2}}}{2 a^{2}},
$$

und, wie sich leicht berechnen lässt

demnach mittelst (2)

$$
A R=\frac{\left(4 a^{4}+r^{4}\right)^{\frac{3}{2}}}{r\left(4 a^{4}+3 r^{4}\right)}
$$

und mittelst (1)

$$
P D=\frac{r\left(4 a^{4}+r^{4}\right)^{\frac{3}{2}}}{2 a^{2}\left(4 a^{4}+3 r^{4}\right)}
$$

$$
x=\frac{r\left(4 a^{4}+r^{4}\right)^{\frac{3}{2}}}{2 a^{2}\left(4 a^{4}-r^{4}\right)}
$$

und, wenn man die Normale $P Q$ mit $N$ bezeichnet:

$$
x=\frac{4 a^{4} N^{3}}{r^{2}\left(4 a^{4}-r^{4}\right)} \text {. }
$$

